Original paper

\title{
Impact of left ventricular diastolic dysfunction on liver transplantation outcomes based on the latest American Society of Echocardiography/ European Association of Cardiovascular Imaging recommendations
}

\author{
Hemnishil K. Marella', Neeraja Yedlapati², Jiten P. Kothadia', Vamsee K. Mupparaju ${ }^{3}$, Saisindhu Marella4 ${ }^{4}$, Satheesh P. Nair ${ }^{1}$ \\ 'Division of Gastroenterology and Hepatology, University of Tennessee Health Science Center, Methodist University Hospital, Memphis, \\ Tennessee, USA \\ ${ }^{2}$ Division of Cardiology, University of Tennessee Health Science Center, Memphis, Tennessee, USA \\ ${ }^{3}$ Methodist Le Bonheur Healthcare, Memphis, Tennessee, USA \\ ${ }^{4}$ University of Louisville, College of Medicine, Louisville, Kentucky, USA
}

\begin{abstract}
Aim of the study: Cirrhotic cardiomyopathy encompasses systolic dysfunction, left ventricular diastolic dysfunction (LVDD), and conduction abnormalities. This study aims to investigate the impact of LVDD on mortality in patients undergoing liver transplantation (LT).

Material and methods: A retrospective review of 400 consecutive patients who underwent LT at our institution was performed. Patient demographics, clinical data, and transthoracic echocardiogram (TTE) were reviewed to identify LVDD. The total cohort consisted of 266 patients after excluding patients with insufficient TTE data $(n=56)$, patients with indeterminate $\operatorname{LVDD}(n=71)$, and patients with ejection fraction $(\mathrm{EF})<55 \%(n=7)$. Statistical analysis was performed using descriptive statistics. Cox regressions with hazard ratios (HRs) and 95\% confidence intervals (CI) were applied to predict 5-year all-cause mortality. Kaplan-Meier survival analysis was conducted to understand the impact of LVDD on 5-year all-cause mortality.

Results: Patients with LVDD have higher incidence of hyperlipidemia ( $36 \%$ vs. $17 \%, p=0.003$ ), hypertension ( $50 \%$ vs. $27 \%, p=0.001$ ) and diabetes ( $52 \%$ vs. $30 \%, p=0.003$ ). In addition, patients with non-alcoholic steatohepatitis (NASH) were more likely to have LVDD ( $48 \%$ vs. $24 \%, p=0.001$ ). A multivariate logistic regression analysis was performed with age, body mass index (BMI), NASH, alcoholic cirrhosis, hepatitis C, history of diabetes, history of hyperlipidemia, and history of hypertension. In this multivariate logistic regression analysis, NASH (odds ratio $[\mathrm{OR}]=4.43[1.10-17.8], p=0.04)$, and history of hypertension (OR $=2.33[1.16-4.66], p=0.01$ ) were independent predictors of LVDD. The Kaplan-Meier survival analysis and multivariate Cox regression demonstrated that the presence of LVDD had no impact on 5-year all-cause mortality (log-rank test nonsignificant).

Conclusions: This study indicates that LVDD in end-stage liver disease (ESLD) patients does not affect immediate post-transplant outcomes or 5-year all-cause mortality.

Key words: left ventricular diastolic dysfunction, non-alcoholic steatohepatitis, cirrhosis, liver transplant, cirrhotic cardiomyopathy.
\end{abstract}

Address for correspondence

Hemnishil K. Marella, D.0., Division of Gastroenterology and Hepatology, University of Tennessee Health Science Center, Methodist University Hospital, 956 Court Avenue Suite H314, Memphis TN, 38163, phone: (502)330-8670,

e-mail: hmarella@uthsc.edu 


\section{Introduction}

Cardiovascular complications are among the leading causes of death after liver transplantation (LT) $[1,2]$. Large studies have revealed that death from cardiovascular disease is the leading cause of early mortality $(40 \%)$, followed by infection (28\%), and graft failure (12\%) [3]. Invasive and noninvasive cardiac tests prior to LT have poor discriminative ability to predict early cardiac mortality after LT [4]. It has been estimated that as many as $50 \%$ of patients undergoing LT developed some signs of cardiac dysfunction, and about $7-21 \%$ of patients died from heart failure in the post-LT period $[5,6]$. Underlying cirrhotic cardiomyopathy (CC) may remain silent and unrecognized with preserved systolic function for a prolonged period. Systolic dysfunction is often masked at rest by the systemic vasodilation and increased heart rate leading to increased cardiac output and is often revealed perioperatively during LT [7]. Additionally, left ventricular diastolic dysfunction (LVDD) is usually the early manifestation with an estimated prevalence ranging from $15 \%$ to $54 \%$ [8-16]. The most common risk factors for LVDD is history of arterial hypertension, diabetes mellitus, coronary artery disease and obesity [17]. LVDD can be due to left ventricular hypertrophy, infiltrative diseases, infarction and fibrosis. Additionally, myocyte relaxation can be altered by intracellular changes [18]. The diagnostic testing used to screen for LVDD such as echocardiography underwent several modifications between 2009 and 2016 [19, 20].

There are conflicting reports on the association between pre-transplant LVDD and post-LT mortality. LVDD association with CC was first described in Montreal at the 2005 World Congress of Gastroenterology (WCOG) [21]. Many of the previous studies have used various echocardiographic measurements and the 2009 American Society of Echocardiography (ASE) criteria for the evaluation of LVDD, which does not apply to the current clinical practice [22]. The most recent joint ASE guidelines (2016) have established a criterion for LVDD, which focuses on screening for elevated left atrial (LA) pressure, which is further reflective of left ventricle (LV) filling pressures [20]. The diagnostic criteria for LVDD that were first established in 2005 at the WCOG rely on parameters that are impacted by loading conditions and heart rate, which can vary significantly in patients with end-stage liver disease (ESLD) $[6,22]$. In this study, we aimed to study the impact of pre-transplant LVDD in general irrespective of etiology, on short-term (30-days) and long-term mortality in ESLD patients undergoing liver transplantation using the 2016 (latest) American

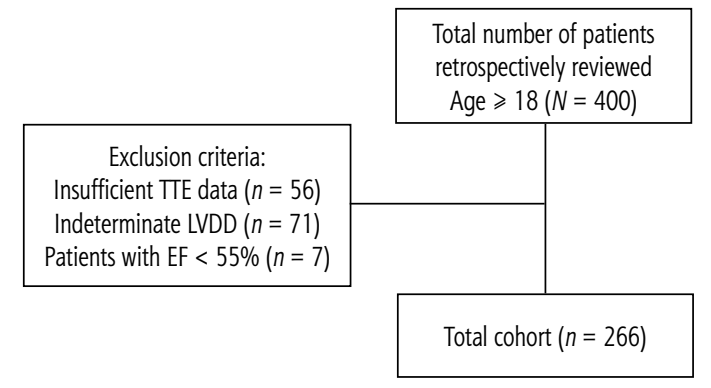

Fig. 1. Depiction of sample selection. Out of 400 patients who underwent liver transplant, 134 patients were excluded based on the criterion mentioned in the figure. Transthoracic echocardiogram (TTE) represents transthoracic echocardiography and left ventricular diastolic dysfunction (LVDD) represents left ventricular diastolic dysfunction

Society of Echocardiography/European Association of Cardiovascular Imaging (EACVI) recommendations.

\section{Material and methods}

\section{Patient characteristics}

We retrospectively reviewed 400 consecutive patients (Fig. 1) who underwent LT at Methodist University Hospital, Memphis, Tennessee, USA. Patients aged 18 years or older were included in the study. The total cohort consisted of 266 patients after excluding patients with insufficient transthoracic echocardiogram (TTE) data $(n=56)$, patients with indeterminate LVDD $(n=71)$, and patients with ejection fraction $(\mathrm{EF})<55 \%$ $(n=7)$. We have not used other criteria to classify these excluded patients to avoid skewing the data. Although LVDD is further classified into grades I, II and III, the power will be too low to compare survival among these groups. Demographic and clinical variables of patients were obtained at the time of hospital admission which included: age, gender, height, weight, body mass index (BMI), sodium, creatinine, albumin, total bilirubin, aspartate aminotransferase (AST), alanine aminotransferase (ALT), alkaline phosphatase, hemoglobin $\mathrm{A}_{1 \mathrm{c}}$, platelet count and international normalized ratio (INR). Patients' comorbidities such as hyperlipidemia, hypertension, diabetes etc. were also recorded. The etiology of cirrhosis was established based on the liver biopsy report before LT and/or the explant liver biopsy report. The severity of liver disease was assessed using the MELD-Na score and was compared between the two groups. The immediate post-operative outcomes were measured, which included: duration of vasopressor therapy, total days on mechanical ventilation, length of intensive care unit (ICU) stay, total length of stay, 30-day all-cause mortality and 5-year all-cause mortality. 


\section{Echocardiography}

Transthoracic echocardiograms were performed based on ASE standards by an experienced sonographer(s) and were read by board-certified cardiologists. TTEs were performed using a GE vivid E95 to evaluate cardiac morphology and function. The TTE reports which were within six months of LT were accessed, and the latest 2016 ASE/EACVI recommendations were used to define and grade the LVDD [20]. The presence or absence of LVDD in patients with a normal LVEF was based on the assessment of four variables. These variables and their cutoff values were: septal e' $<7 \mathrm{~cm} / \mathrm{s}$ or lateral e' $<10 \mathrm{~cm} / \mathrm{s}$, average E/e' $>14$, LA volume in$\operatorname{dex}\left(\right.$ LAVI) $>34 \mathrm{ml} / \mathrm{m}^{2}$, and peak tricuspid regurgitation (TR) velocity $>2.8 \mathrm{~m} / \mathrm{s}$. Left ventricular diastolic function is normal if more than half of the available variables are normal ( $<50 \%$ positive), LVDD is present if more than half of the available variables are abnormal (> 50\% positive) and in cases in which half of the variables do not meet the cutoff value, the study is indeterminate (50\% positive) [20].

\section{Statistical analysis}

Statistical analysis was performed using descriptive statistics; 2-tailed unpaired $t$-test, Fisher's exact test and $\chi^{2}$ test were applied as appropriate for continuous and categorical data. Patient characteristics were summarized as means and standard deviation. A $p$-value $<0.05$ was considered statistically significant. Multivariate logistic regression analysis was conducted to identify predictors of LVDD. Cox regressions with hazard ratios (HRs) and 95\% confidence interval (CI) were applied for the prediction of mortality. We then computed the adjusted HRs and 95\% CIs to estimate the strength of association of each predictor and its association with all-cause mortality. Kaplan-Meier survival analysis was conducted to evaluate the impact of LVDD on 5-year all-cause mortality. Data were analyzed using STATA 15 (StataCorp, College Station, TX).

\section{Results}

After excluding patients based on the criteria described above, the final cohort consisted of 266 con-

Table 1. Patient characteristics and clinical outcomes based on left ventricular diastolic dysfunction

\begin{tabular}{|c|c|c|c|c|c|c|c|}
\hline Clinical characteristics & $\begin{array}{c}\text { Diastolic } \\
\text { dysfunction } \\
(n=50)\end{array}$ & $\begin{array}{c}\text { No diastolic } \\
\text { dysfunction } \\
(n=216)\end{array}$ & $p$ value & Clinical characteristics & $\begin{array}{c}\text { Diastolic } \\
\text { dysfunction } \\
(n=50)\end{array}$ & $\begin{array}{c}\text { No diastolic } \\
\text { dysfunction } \\
(n=216)\end{array}$ & $p$ value \\
\hline Age (years), mean $\pm S D$ & $59.5 \pm 9.3$ & $55.2 \pm 10.6$ & $0.009^{\#}$ & MELD-Na, mean \pm SD & $22.4 \pm 7.8$ & $23.0 \pm 8.2$ & $0.67^{\#}$ \\
\hline Gender (female \%) & $20(40)$ & $113(52)$ & $0.117^{*}$ & $\mathrm{HgA}_{1 c^{\prime}}$ mean $\pm \mathrm{SD}$ & $5.6 \pm 1.44$ & $5.1 \pm 1.19$ & $0.07^{\#}$ \\
\hline BMl $\left(\mathrm{kg} / \mathrm{m}^{2}\right)$, mean $\pm \mathrm{SD}$ & $30.1 \pm 6.6$ & $29.7 \pm 7.1$ & $0.72^{\#}$ & $\mathrm{QTC}$, mean $\pm \mathrm{SD}$ & $435 \pm 29.2$ & $435 \pm 35.3$ & $0.96^{\#}$ \\
\hline Etiology of cirrhosis & & & & \multicolumn{4}{|l|}{ Outcome measures } \\
\hline $\mathrm{NASH}, n(\%)$ & $24(48)$ & $52(24)$ & $0.001^{*}$ & \multirow{2}{*}{$\begin{array}{l}\text { Length of ICU stay (days), } \\
\text { mean } \pm \text { SD }\end{array}$} & \multirow[t]{2}{*}{$3.82 \pm 2.37$} & \multirow[t]{2}{*}{$4.66 \pm 6.61$} & \multirow[t]{2}{*}{$0.39^{\#}$} \\
\hline Hepatitis C, n (\%) & $15(30)$ & $73(34)$ & $0.61^{*}$ & & & & \\
\hline Alcohol, $n(\%)$ & $8(16)$ & $48(22)$ & $0.331^{\wedge}$ & \multirow{2}{*}{$\begin{array}{l}\text { Overall length of stay (days), } \\
\text { mean } \pm \text { SD }\end{array}$} & \multirow[t]{2}{*}{$14.2 \pm 9.11$} & \multirow[t]{2}{*}{$14.6 \pm 12.8$} & \multirow[t]{2}{*}{$0.85^{\#}$} \\
\hline PBC, $n(\%)$ & $0(0)$ & $1(0.5)$ & $1.00^{\wedge}$ & & & & \\
\hline PSC, $n(\%)$ & $0(0)$ & $6(3)$ & $0.598^{\wedge}$ & $\begin{array}{l}\text { Days on vasopressors, } \\
\text { mean } \pm S D\end{array}$ & $0.20 \pm 0.91$ & $0.47 \pm 2.28$ & $0.41^{\#}$ \\
\hline Autoimmune, $n(\%)$ & $2(4)$ & $15(7)$ & $0.748^{\wedge}$ & \multirow{2}{*}{$\begin{array}{l}\text { Days on mechanical } \\
\text { ventilation, mean } \pm S D\end{array}$} & \multirow{2}{*}{$1.41 \pm 1.23$} & \multirow{2}{*}{$2.31 \pm 5.9$} & \multirow{2}{*}{$0.30^{\#}$} \\
\hline Other, $n(\%)$ & $1(2)$ & $20(9)$ & $0.141^{\wedge}$ & & & & \\
\hline Comorbidities & & & & Cardiac arrhythmias, $n(\%)$ & $2(4)$ & $10(5)$ & $1.00^{\wedge}$ \\
\hline $\begin{array}{l}\text { History of hyperlipidemia, } \\
n(\%)\end{array}$ & $18(36)$ & $37(17)$ & $0.003^{*}$ & $\begin{array}{l}\text { Cardiac adverse events**, } \\
n(\%)\end{array}$ & $2(4)$ & $10(5)$ & $1.00^{\wedge}$ \\
\hline $\begin{array}{l}\text { History of hypertension, } \\
n(\%)\end{array}$ & $25(50)$ & $58(27)$ & $0.001^{*}$ & $\begin{array}{l}\text { 30-day all-cause mortality, } \\
n(\%)\end{array}$ & $4(8)$ & $15(7)$ & $0.763^{\wedge}$ \\
\hline History of diabetes, $n(\%)$ & $26(52)$ & $65(30)$ & $0.003^{*}$ & \multirow{2}{*}{$\begin{array}{l}\text { 5-year all-cause mortality, } \\
n(\%)\end{array}$} & \multirow[t]{2}{*}{$8(16)$} & \multirow[t]{2}{*}{$37(17)$} & \multirow[t]{2}{*}{$0.7682^{\prime}$} \\
\hline History of smoking, $n(\%)$ & $9(18)$ & $47(22)$ & $0.557^{\wedge}$ & & & & \\
\hline
\end{tabular}

${ }^{*} \chi^{2}$, \#2-tailed unpaired t-test,

${ }^{\wedge}$ Fisher's exact test, $\mathrm{HgA}$, was available in 172 patients

* *Cardiac complications: cardiac arrest, myocardial infarction, cardiomyopathy, heart failure exacerbation 
secutive patients who underwent LT. The patient characteristics are shown in Table 1. The cohort consisted of $50(18.7 \%)$ patients with pre-transplant LVDD. The prevalence of grade 1 LVDD was $9(18 \%)$, grade 2 LVDD 39 (78\%), grade 3 LVDD 2 (4\%). Patients with LVDD were older (59.5 years vs. 55.2 years, $p=0.009$ ). The most common reason for $\mathrm{LT}$ was hepatitis $\mathrm{C}$ virus (HCV) 88 (33\%), followed by non-alcoholic steatohepatitis (NASH) $76(28.5 \%)$ and alcoholic liver disease 56 (21\%). Patients with NASH were more likely to have LVDD [24 (48\%) vs. 52 (24\%), $p=0.001$ ]. In addition, patients with LVDD had a higher incidence of hyperlipidemia [ $18(36 \%)$ vs. $37(17 \%), p=0.003$ ], hypertension $[25(50 \%)$ vs. $58(27 \%), p=0.001]$ and diabetes [26 (52\%) vs. 65 (30\%), $p=0.003$ ]. The mean MELD-Na was similar between the LVDD and the non-LVDD group (22.4 vs. 23.0, $p=0.67$ ). The mean QTC was similar between the LVDD and the non-LVDD group (435 \pm 29.2 vs. $435 \pm 35.3, p=0.96$ ). Univariate analysis was done with each variable and those variables with significance were included in the multivariate analysis. A multivariate logistic regression analysis was performed with age, BMI, NASH, alcoholic cirrhosis, cirrhosis secondary to HCV, history of diabetes, history of hyperlipidemia and history of hypertension. In multivariate logistic regression analysis, NASH (odds ratio $[\mathrm{OR}]=4.43[1.10-17.8], p=0.04)$ and history of hypertension $(\mathrm{OR}=2.33$ [1.16-4.66], $p=0.01$ ) were independent predictors of LVDD.

Outcomes of patients with and without LVDD are shown in Table 1. The incidence of cardiac arrhythmias, incidence of cardiac adverse events (i.e. cardiac arrest, myocardial infarction, cardiomyopathy, heart failure exacerbation) in the immediate post-operative period or 30-day mortality was not statistically different between the two groups. There was no statistically significant difference observed in the length of ICU stay, the total length of hospital stay, the number of days on mechanical ventilation or the number of days on vasopressors between the two groups. Over 5 years, there were 45 deaths in this cohort, eight patients with LVDD, and 37 patients without LVDD. The Kaplan-Meier 5-year survival analysis (Fig. 2) demonstrated that LVDD had no impact on 5-year all-cause mortality (log-rank test non-significant). A multivariable Cox regression survival model was conducted with age, BMI, gender, LVDD and etiologies of cirrhosis such as HCV, NASH, and alcohol. In this final multivariable Cox regression survival model, LVDD was not an independent predictor of 5-year all-cause mortality. However, BMI ( $\mathrm{HR}=1.05$ [95\% 1.0-1.10], $p=0.027)$, ESLD secondary to $\mathrm{HCV}(\mathrm{HR}=4.00[95 \%$ $1.10-23.9], p=0.04)$ and NASH $(\mathrm{HR}=5.77[95 \% 1.23$ -
$27.1], p=0.026)$ were the only independent predictors of 5 -year all-cause mortality.

\section{Discussion}

Left ventricular diastolic dysfunction is considered an early marker of cardiac dysfunction occurring before systolic dysfunction and believed to impact mortality in post-transplant patients. In previous studies, the prevalence of LVDD ranged from $15 \%$ to $54 \%$ in ESLD patients [8-16]. Our results showed that the prevalence of pre-transplant LVDD was 19\% in transplant recipients. Also, the presence of pre-transplant LVDD did not adversely impact the immediate transplant outcome or long-term 5-year all-cause mortality. Higher BMI, ESLD secondary to HCV, and NASH were the independent predictors of 5-year all-cause mortality.

This variable prevalence of LVDD in prior studies is likely due to the wide array of criteria that have been used to define LVDD including the 2005 WCOG criteria, 2009 ASE and European Association of Echocardiography criteria, and also using the individual echocardiography parameters such as left atrial volume index, E/A ratio $<1, \mathrm{E} / \mathrm{E}^{\prime}>10$ and left ventricular mass index (LVMI) $>40$ [9-11]. The 2005 WCOG defined LVDD as a decreased $\mathrm{E} / \mathrm{A}$ ratio, increased deceleration time, and increased isovolumetric relaxation time, which indicates delayed relaxation and is a non-specific phenomenon $[19,20]$. The most recent (2016) ASE/EACVI guidelines use four recommended variables for identifying LVDD, which are annular $e^{\prime}$ velocity: septal e' $(<7 \mathrm{~cm} / \mathrm{s})$ or lateral e' $(<10 \mathrm{~cm} / \mathrm{s})$, average E/e' ratio $(>14)$, and LAVI $\left(>34 \mathrm{ml} / \mathrm{m}^{2}\right)$ and tricuspid valve regurgitation (TR) velocity $(>2.8 \mathrm{~m} / \mathrm{s})$. If $\geq 3$ variables are abnormal, LVDD is present. The

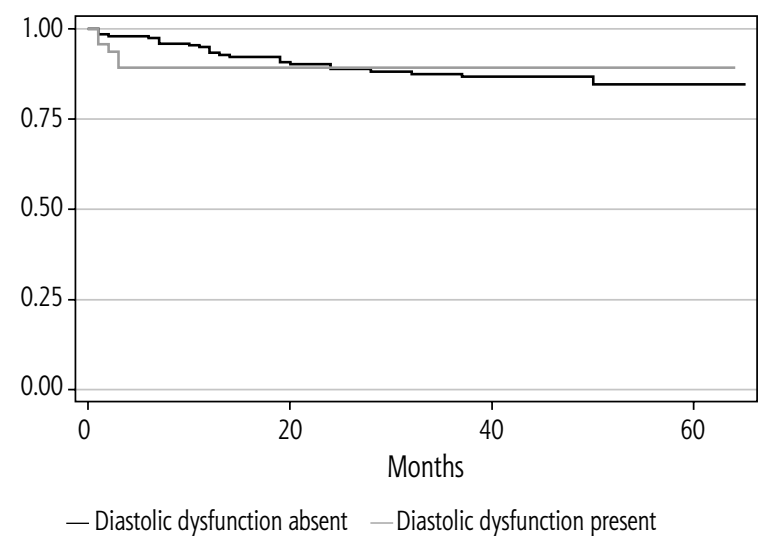

Log rank test $p=0.7682$

Fig. 2. Five-year survival analysis. Kaplan-Meier 5-year survival analysis comparing patients with and without left ventricular diastolic dysfunction showed no statistically significant difference between the two groups 
$\mathrm{E} / \mathrm{A}$ ratio determines its severity or grading of LVDD: grade 3 when $\mathrm{E} / \mathrm{A} \geq 2$ and grade 2 when $\mathrm{E} / \mathrm{A}<2$ [20]. Our study utilized this latest criterion, allowing us to evaluate the applicability of these criteria in determining the presence or absence of LVDD in cirrhotics, in turn helping us to understand the impact of LVDD on ESLD patients undergoing LT. The 2016 ASE criteria were most recently endorsed by the Cirrhotic Cardiomyopathy Consortium [23]. The criteria were modified to identify LVDD with more specificity to detect increased left atrial pressure, which is reflective of elevated pulmonary capillary wedge pressure (PCWP) $>15 \mathrm{mmHg}$ and is the gold standard for clinical diagnosis of LVDD [23].

As stated previously, our study noted that the presence of pre-transplant LVDD did not adversely affect the immediate transplant outcome or 5-year all-cause mortality. Previous studies have yielded conflicting reports on the impact of LVDD. It is important to note that these studies have utilized different criteria for defining LVDD or used individual echocardiographic parameters to study the outcomes. These studies show that left ventricular hypertrophy (LAVI $>40)$, E/E' $>10$ and $\mathrm{E} / \mathrm{A}<1$ are associated with post-transplantation mortality [9-11]. Additionally, Carvalherio et al. [8] used the E/A ratio (corrected for age), prolonged deceleration time (>200 ms), and prolonged isovolumetric relaxation time (> $80 \mathrm{~ms})$, and annular E' and E/E' lateral ratio to define LVDD. They noted that LVDD was associated with mortality in cirrhotic patients undergoing LT. In addition, Karagiannakis et al. [12] had a small sample size of 45 patients and defined LVDD based on the 2009 ASE criteria for LVDD and found that LVDD may lead to poor prognosis. Mittal et al. [13] used the E/A ratio and $\mathrm{E} / \mathrm{E}$ ' ratio to define LVDD among 970 patients. In this study grade 2 and/ or grade 3 LVDD was associated with higher graft failure and mortality. Additionally, Ruiz-del-Arbol et al. [15] demonstrated that LVDD is a sensitive marker for advanced fibrosis, type 1 hepatorenal syndrome (HRS) development, and mortality.

In contrast to those studies, Raevens et al. [14] reported no association of LVDD with mortality using $\mathrm{E} / \mathrm{A}<1$ and deceleration time $>200$ milliseconds. Sonny et al. [16] found that the prevalence of LVDD was 54\% in their cohort based on the 2009 ASE criteria. These patients were followed for approximately five years, and it was concluded that LVDD did not impact outcomes. As the criteria for LVDD evolves, it is essential to understand its relevance in the current patient population. This was supported by the study of Park et al. [22], which showed that the 2016 ASE/EACVI guidelines more accurately identify the patients at risk for LVDD in LT compared to the 2009 ASE criteria. Additionally, the 2020 Cirrhotic Cardiomyopathy Consortium endorsed the 2016 ASE criteria for defining LVDD with a small modification [23].

Limitations of our study are inherent to the retrospective design, as it may be affected by inter-observer variability in the TTE reports. We excluded almost 56 patients due to incomplete LVDD echocardiogram criteria and 71 patients with indeterminate LVDD. However, this should not affect our results, as these patients were excluded from the study. The findings of this study need to be replicated in a large multicenter study. In conclusion, our study highlights that LVDD in pre-transplant patients does not affect short-term or long-term post-transplant outcomes.

\section{Disclosure}

The authors declare no conflict of interest.

\section{References}

1. Liu H, Lee SS. Predicting cardiovascular complications after liver transplantation: 007 to the rescue? Liver Transpl 2011; 17: 7-9.

2. Watt KD, Pedersen RA, Kremers WK, et al. Evolution of causes and risk factors for mortality post-liver transplant: results of the NIDDK long-term follow-up study. Am J Transplant 2010; 10: 1420-1427.

3. VanWagner LB, Lapin B, Levitsky J, et al. High early cardiovascular mortality after liver transplantation. Liver Transpl 2014; 20: 1306-1316.

4. Raval Z, Harinstein ME, Skaro AI, et al. Cardiovascular risk assessment of the liver transplant candidate. J Am Coll Cardiol 2011; 58: 223-231.

5. Myers RP, Lee SS. Cirrhotic cardiomyopathy and liver transplantation. Liver Transpl 2000; 6: S44-52.

6. Zardi EM, Abbate A, Zardi DM, et al. Cirrhotic cardiomyopathy. J Am Coll Cardiol 2010; 56: 539-549.

7. Liu H, Jayakumar S, Traboulsi M, et al. Cirrhotic cardiomyopathy: Implications for liver transplantation. Liver Transpl 2017; 23: 826-835.

8. Carvalheiro F, Rodrigues C, Adrego T, et al. Diastolic dysfunction in liver cirrhosis: prognostic predictor in liver transplantation? Transplant Proc 2016; 48: 128-131.

9. Darstein F, Konig C, Hoppe-Lotichius M, et al. Preoperative left ventricular hypertrophy is associated with reduced patient survival after liver transplantation. Clin Transplant 2014; 28 : 236-242.

10. Dowsley TF, Bayne DB, Langnas AN, et al. Diastolic dysfunction in patients with end-stage liver disease is associated with development of heart failure early after liver transplantation. Transplantation 2012; 94: 646-651.

11. Josefsson A, Fu M, Allayhari P, et al. Impact of peri-transplant heart failure \& left-ventricular diastolic dysfunction on outcomes following liver transplantation. Liver Int 2012; 32: 1262-1269. 
12. Karagiannakis DS, Vlachogiannakos J, Anastasiadis G, et al. Diastolic cardiac dysfunction is a predictor of dismal prognosis in patients with liver cirrhosis. Hepatol Int 2014; 8: 588-594.

13. Mittal C, Qureshi W, Singla S, et al. Pre-transplant left ventricular diastolic dysfunction is associated with post transplant acute graft rejection and graft failure. Dig Dis Sci 2014; 59: 674-680.

14. Raevens S, De Pauw M, Geerts A, et al. Prevalence and outcome of diastolic dysfunction in liver transplantation recipients. Acta Cardiol 2014; 69: 273-280.

15. Ruiz-del-Arbol L, Achecar L, Serradilla R, et al. Diastolic dysfunction is a predictor of poor outcomes in patients with cirrhosis, portal hypertension, and a normal creatinine. Hepatology 2013; 58: 1732-1741.

16. Sonny A, Ibrahim A, Schuster A, et al. Impact and persistence of cirrhotic cardiomyopathy after liver transplantation. Clin Transplant 2016; 30: 986-993.

17. Yancy CW, Jessup M, Bozkurt B, et al. 2013 ACCF/AHA guideline for the management of heart failure: a report of the American College of Cardiology Foundation/American Heart Association Task Force on Practice Guidelines. J Am Coll Cardiol 2013; 62: e147-239.

18. Wan SH, Vogel MW, Chen HH. Pre-clinical diastolic dysfunction. J Am Coll Cardiol 2014; 63: 407-416.

19. Nagueh SF, Appleton CP, Gillebert TC, et al. Recommendations for the evaluation of left ventricular diastolic function by echocardiography. J Am Soc Echocardiogr 2009; 22: 107-133.

20. Nagueh SF, Smiseth OA, Appleton CP, et al. Recommendations for the evaluation of left ventricular diastolic function by echocardiography: an update from the American Society of Echocardiography and the European Association of Cardiovascular Imaging. J Am Soc Echocardiogr 2016; 29: 277-314.

21. Moller S, Henriksen JH. Cardiovascular complications of cirrhosis. Gut 2008; 57: 268-278.

22. Park J, Lee J, Kwon A, et al. The 2016 ASE/EACVI recommendations may be able to more accurately identify patients at risk for diastolic dysfunction in living donor liver transplantation. PLoS One 2019; 14: e0215603.

23. Izzy M, VanWagner LB, Lin G, et al. Redefining cirrhotic cardiomyopathy for the modern era. Hepatology 2020; 71: 334-345. 